\title{
Síndrome de cimitarra, valoración con ecocardiografía transesofágica intraoperatoria. Informe de caso
}

\section{Scimitar syndrome; intraoperative transesophageal echocar- diography assessment.Case report}

Walter Damián Rodríguez', Silvina Longo², Maximiliano Dohmen. ${ }^{1}$

\begin{abstract}
9 year old patient diagnosed with scimitar syndrome through transthoracic echocardiogram and cardiac catheterization. Surgical correction is performed by anastomosing the collector of the pulmonary right vein to Al through the interatrial septum, tunneling it with a pericardic patch. The study findings and echocardiographic images of such surgical repair and the spectrum of pulsed Doppler differentiating between right and left pulmonary veins will be shown below. The connection through the septum was evidenced, simulating an interatrial communication. A difference was found among the measured points $S, D, A$ and between the left and right pulmonary veins, without this resulting in a stenosis during the repair.
\end{abstract}

\section{RESUMEN}

Paciente de 9 años de edad el cual es diagnosticado con síndrome de cimitarra a través de ecografía transtorácica y cateterismo cardiaco. Se realiza cirugía correctora la cual se anastomosa el colector de vena pulmonar derecha hacia Al a través del septum interauricular, tunelizándolo con parche pericárdico. A continuación se muestran los hallazgos e imágenes ecocardiográficas de tal reparación y el espectro doppler pulsado diferenciado entre las venas pulmonares derechas e izquierdas. Se pudo evidenciar la conexión a través del septum, simulando una comunicación interauricular. Se halló una diferencia entre los puntos medidos $S$, $\mathrm{D}, \mathrm{A}$ entre las venas pulmonares izquierdas y derechas, sin que esto significara una estenosis en la reparación.

\section{Key words:}

Scimitar syndrome, echocardiography, surgical correction

\section{Palabras clave:}

Síndrome cimitarra, ecocardiografía, poscorrección

Servicio de Anestesiología; Hospital Pediátrico Humberto Notti. Mendoza, Argentina.

Servicio de Anestesiología; Hospital Privado Universitario de Córdoba, Argentina.

Fecha de recepción: 29 de agosto de 2018

Fecha de aceptación: 22 de septiembre de 2018

\section{ORCID}

https://orcid.org/0000-0003-4133-0623 


\section{Introducción}

E I síndrome de cimitarra es una rara patología en su frecuencia. Cerca de 3-6\% de las anomalías parciales de retorno venoso pulmonar[1] y el 05$2 \%$ de las cardiopatías congénitas[6]. Fue descripta por primera vez en 1836 por G. Cooper[2], no tiene predisposición genética ni racial y es más frecuente en el sexo femenino[3].

Probablemente se presenta por alteraciones en la embriogénesis por 1) persistencia, en el lugar de la obliteración normal de la comunicación primitiva entre la aorta y el plexo vascular pulmonar y 2) obliteración prematura de la vena pulmonar común y persistencia de la vía de drenaje primitiva del pulmón en la vena cava inferior[2].

Se lo reconoce como un síndrome desde 1960 presentando: drenaje venoso anómalo de venas pulmonares derechas a la vena cava inferior, hipoplasia del lóbulo inferior derecho del pulmón y de la arteria pulmonar derecha con una anatomía traqueobronquial anómala, irrigación sistémica arterial anómala del lóbulo inferior derecho directamente desde la aorta o de sus ramas principales y dextroposición cardíaca[5].

La sintomatología y gravedad del cuadro depende de la asociación con otras patologías cardíacas (insuficiencia cardiaca por cortocircuito izquierda-derecha, hipertensión pulmonar, alteraciones del crecimiento, distress respiratorio e infecciones respiratorias recurrentes)[3]. Los niños y los adultos pueden presentarse oligosintomáticos (sin insuficiencia cardiaca ni hipertensión pulmonar), con soplos cardíacos o infecciones respiratorias recurrentes e incluso asintomático en los cuales el diagnóstico es un hallazgo casual en una radiografía de rutina[3]-[7]. La indicación o no de cirugía va a depender de los defectos del septo arterial, la HTP o la estenosis de la vena anómala[8].

\section{Paciente e historia hospitalaria}

Se trata de un paciente de sexo masculino, de nueve años de edad, oriundo de la provincia de Mendoza, Argentina. El cual a los 15 días de nacido (16-3-2008) es internado por primera vez con diagnóstico de neumonía, donde por valoración ecográfica se sospecha la anomalía parcial del retorno venoso pulmonar derecho. Se realiza cateterismo y TAC 3D donde se confirma el diagnóstico. Luego de una buena evolución clínica de la patología respiratoria se decide dar el alta con furosemida y programar el turno quirúrgico en dos meses. El paciente no volvió a los controles pautados, regresando a los 5 años de edad, por lo que se repite el cateterismo y se ratifica el diagnóstico. En los siguientes años hubo dos intentos de programar el acto quirúrgico, pero por infecciones respiratorias debieron suspenderse y luego se trasladaron a su lugar de residencia fuera de la provincia, hasta que en 2018 se interna y realiza el acto quirúrgico.

\section{Evaluación diagnóstica}

La vena en cimitarra, generalmente, drena hacia la porción hepática de la cava inferior[6], tal es el caso de nuestro paciente (Figura 1). En el segundo cateterismo realizado (2013), a los 5 años de edad, se produce el hallazgo de una colateral directa desde aorta tóracoabdominal a lóbulo pulmonar inferior derecho de tamaño grande (arteria sistémica anómala) y en
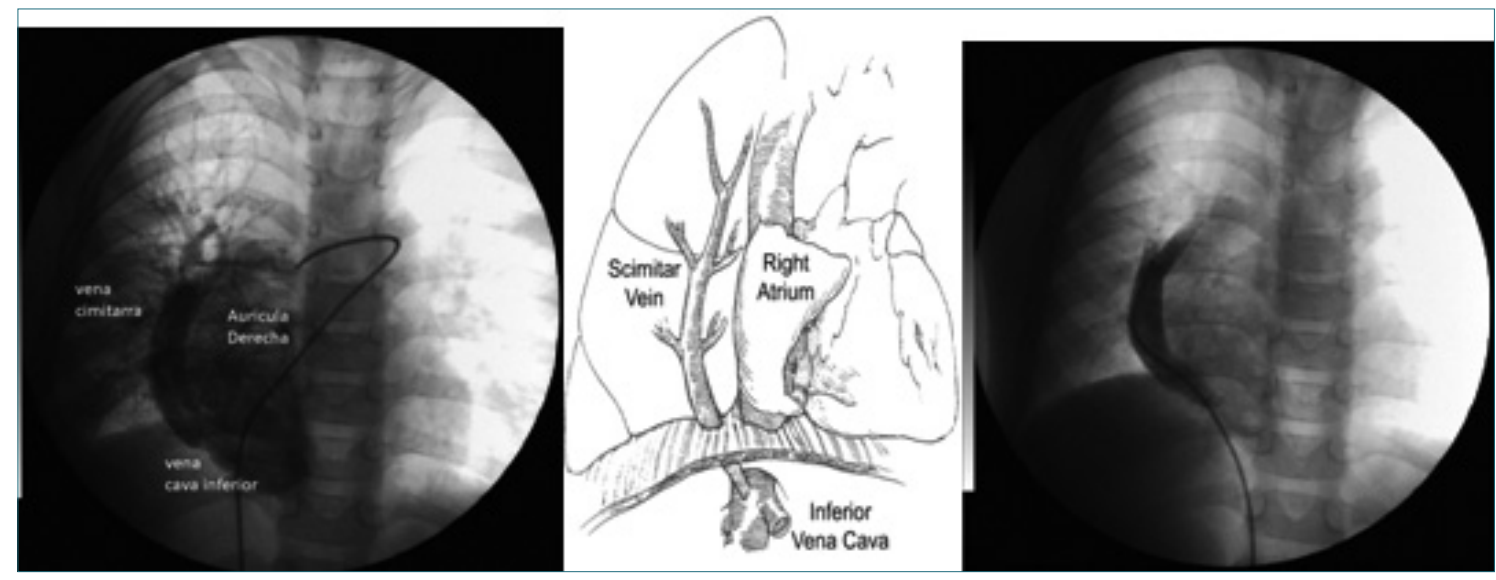

Figura 1. Imágen radioloógica y esquema que muestra el tipo de malformación. 


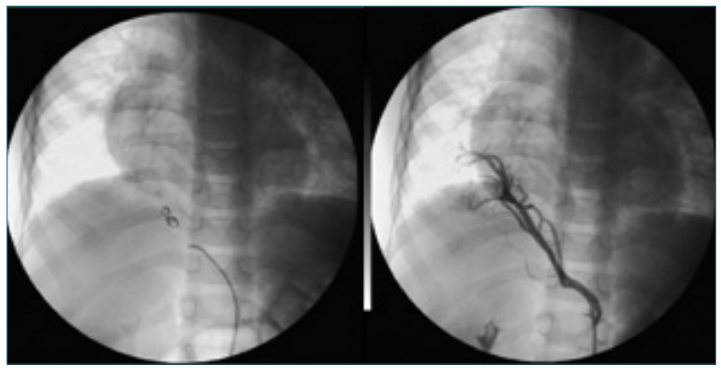

Figura 2. Cierre con coil.

el mismo acto se procede al cierre con coil flipper quedando un shunt residual inmediato mínimo. Este hallazgo es algo característico que se asocia a este síndrome[5] (Figura 2).

\section{Intervención terapéutica}

Ya confirmado él diagnóstico, se realizó la cirugía. La inducción y mantenimiento fue por remifentanilo endovenoso y sevofluorane inhalatorio, con concentraciones de oxígeno de 0,3 y ventilación protectora de atelectasias sin inconvenientes. En el acto quirúrgico, luego de la instauración de circulación extracor- pórea, clampeo y solución cardiopléjica sin percances, los cirujanos comienzan la inspección del septum (intacto) con drenaje de colector de venas pulmonares derechas en vena cava inferior. Se realiza comunicación interauricular y se coloca parche de pericardio tunelizando la vena pulmonar anómala hacia la aurícula izquierda.

En el inicio de la cirugía el primer plano tomado de 4 cámaras, se observa una imagen anexa al VD que simulaba una cámara. Se le aplicó doppler pulsado que evidenció un espectro de flujo venoso, por lo que se supuso que era la vena cava inferior dilatada. Esto se confirmó con la disección quirúrgica (Figura 3). La dilatación también se observaba en venas suprahepáticas e intrahepáticas (Figura 4).

En el desclampeo y comenzado de carga ventricular se comenzó a visualizar el parche de tunelización a través de la CIA (Figura 5).

En la salida de apoyo circulatorio hubo buena tolerancia hemodinámica con muy bajos requerimientos de drogas inotrópicas. Se realizó maniobra de reclutamiento pulmonar la cual finalizó con parámetros respiratorios presión inspiratoria sobre PEEP: 10; PEEP: 4. FR: 15/min $\mathrm{FiO}_{2}$ 0,3\%.

Luego de la salida de bomba se visualizó y aplicó doppler pulsado en la desembocadura de la tuneli-

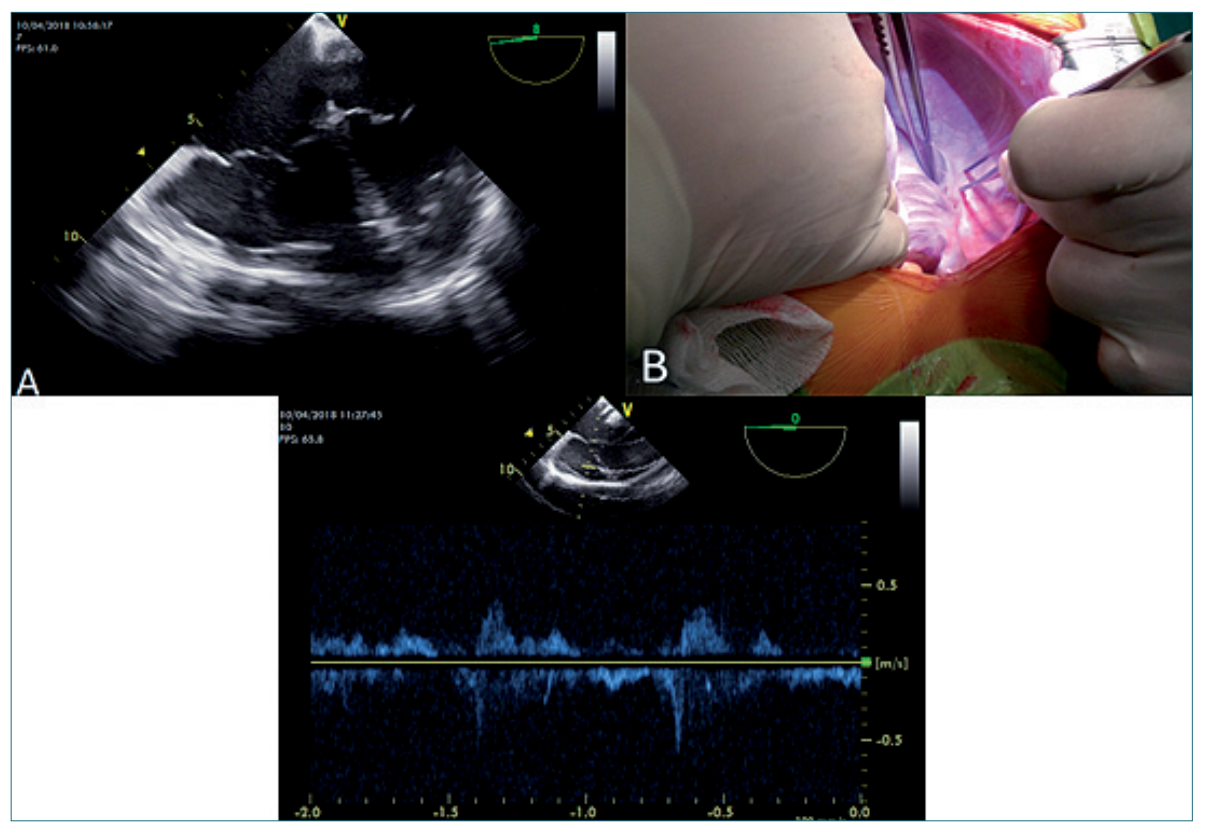

Figura 3. A) imagen de cámara anexa; B) en planos de disección vena cava inferior dilatada. C) espectro doppler. Ver video 1: https://vimeo.com/320255601 Ver video 2: https://vimeo.com/320257709 


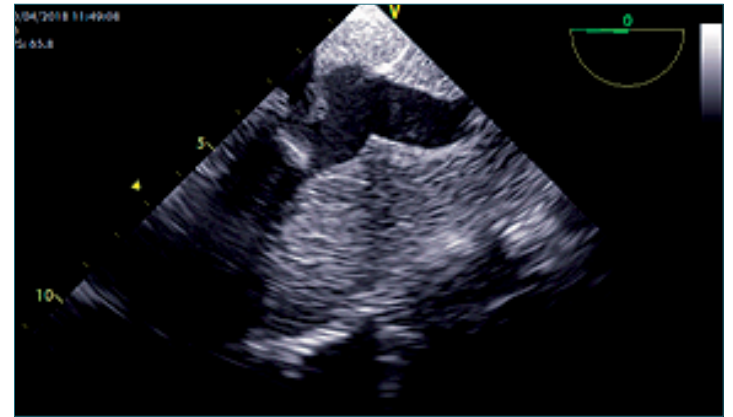

Figura 4. Ver video: https://vimeo.com/320258339

zaión y se comparó con las venas pulmonares izquierdas (Figura 6).

En el análisis del espectro doppler pulsado se encontró una mayor velocidad en la anastomosis (Figura 7a) donde $\mathrm{S}: 1,05 \mathrm{~m} / \mathrm{s} ; \mathrm{D}: 0,8 \mathrm{~m} / \mathrm{s}$ y A: 0,81 m/s. Con respecto al retorno venoso normal (Figura $7 b) \mathrm{S}: 0,75$ $\mathrm{m} / \mathrm{s} ; \mathrm{D}: 0,55 \mathrm{~m} / \mathrm{s} ; A: 0,3 \mathrm{~m} / \mathrm{s}$. El índice S/D fue igual en

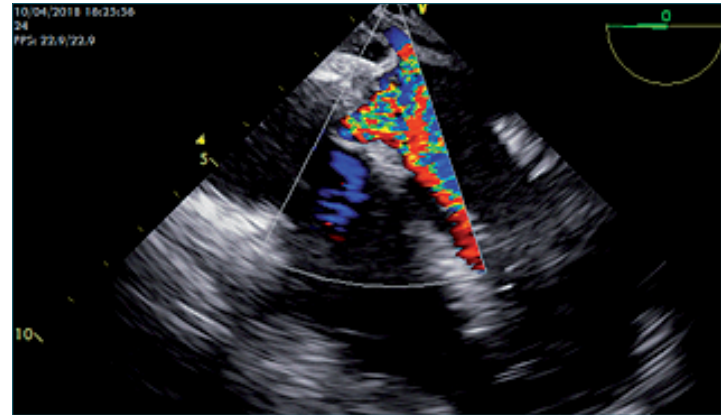

Figura 6. Ver video: https://vimeo.com/320259033

los dos: 1,31 en la anastomosis y 1,37 en la vena normal. Esto lo interpretamos como una consecuencia de una boca anastomótica ancha. Para corroborarlo tomamos el tiempo de la onda S y D para llegar al pico de velocidad y de éste para llegar a su mínimo valor (Figuras 8, 9, 10 y 11). Los tiempos medidos fueron considerablemente mayor (por ejemplo 111 ms vs 37
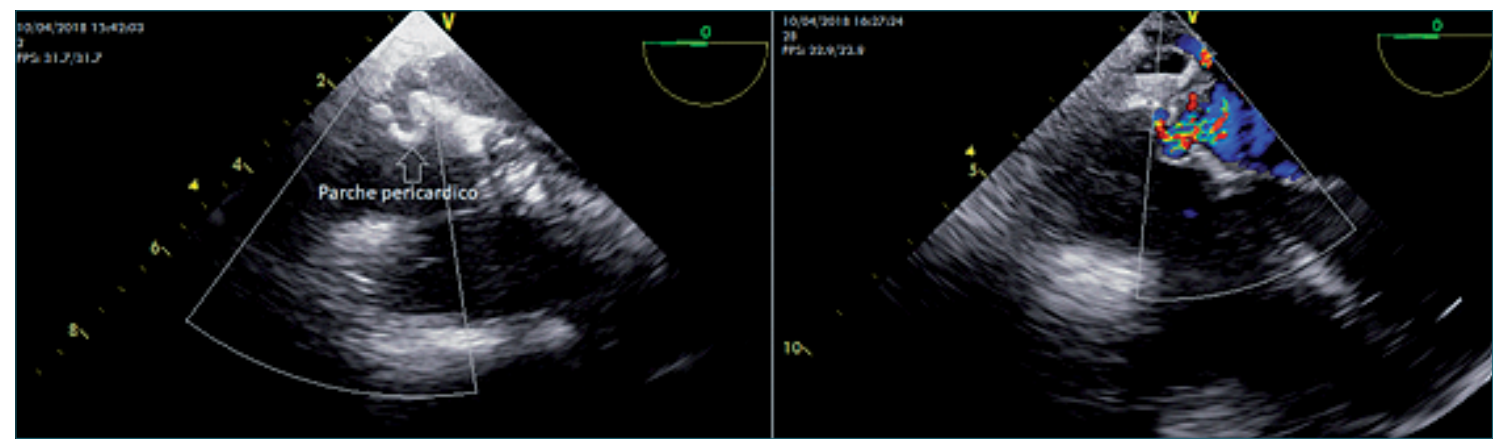

Figura 5. Doppler color del defecto residual.

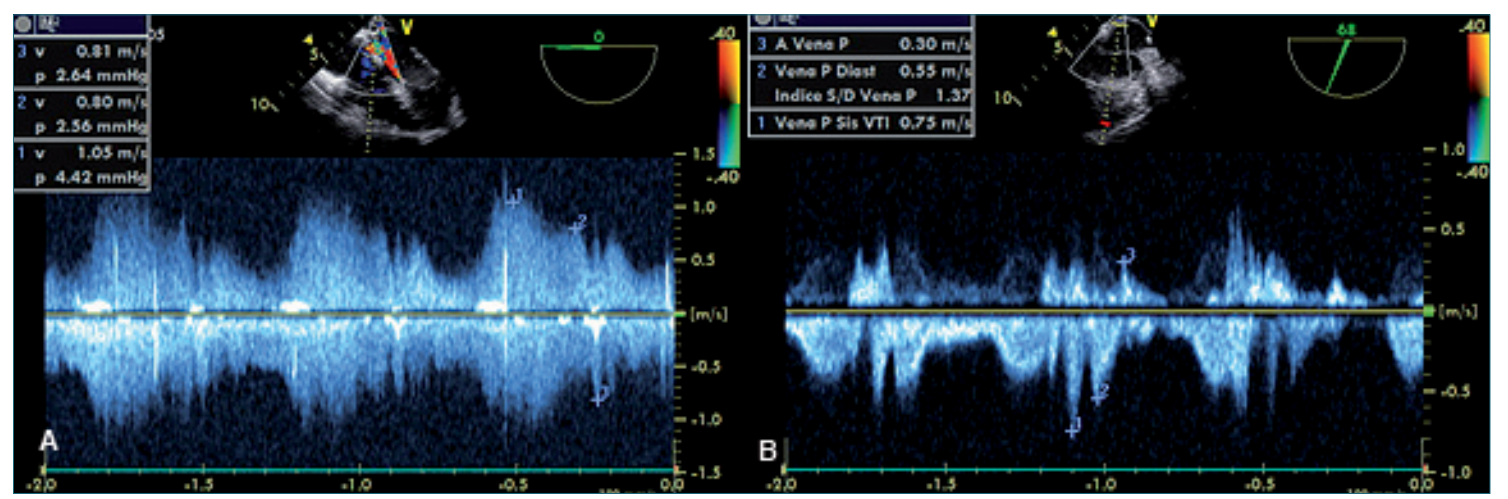

Figura 7. a) doppler de la boca anastomótica; b) Drenaje de las venas pulmonares normales. 


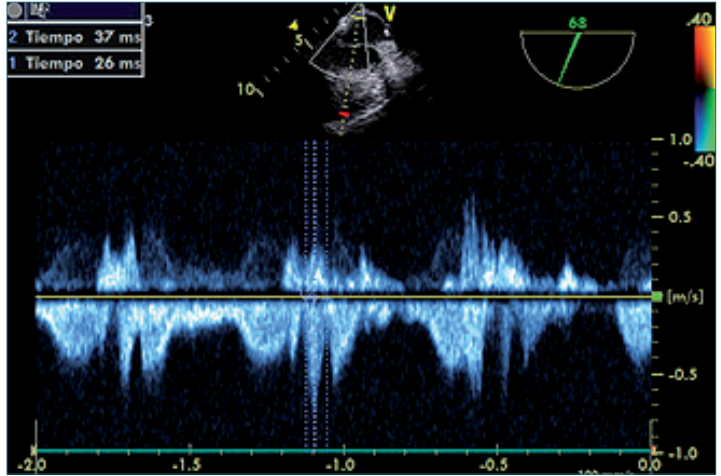

Figura 8. 1) Tiempo para llegar al pico de gradiente; 2) Tiempo para llegar a cero. Onda S. Drenaje normal.

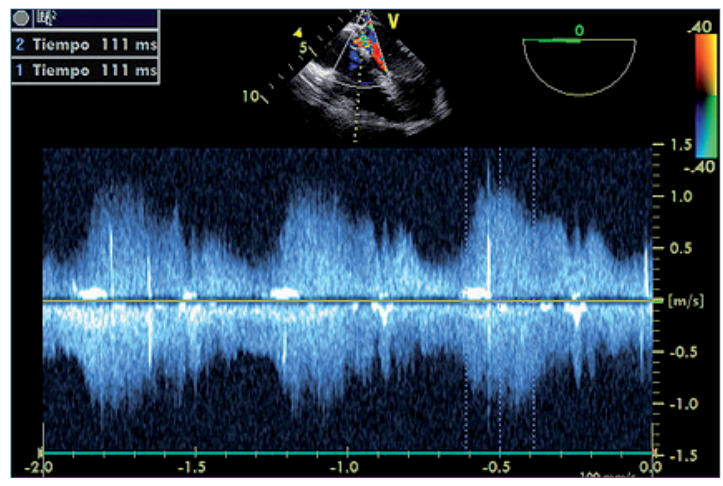

Figura 10. Tiempo para llegar al pico de gradiente; 2 : Tiempo para llegar a cero. Onda S. Drenaje boca anastomótica.

ms). De existir estenosis encontraríamos solo mayor velocidad en menor o igual tiempo.

\section{Planos}

La anastomosis en el septum interauricular se obtuvo en plano medio esofágico a $0^{\circ}$ y se roto ligeramente hacia la derecha para alinear lo más posible el flujo, por lo tanto se obtuvo onda doppler positiva. La otra vena pulmonar derecha pudo encontrarse a $68^{\circ}$ y se obtuvo doppler negativo.

\section{Discusión}

Los síntomas (infecciones respiratorias recurrentes) y métodos diagnósticos (ecocardiografía y cateterismo) fueron similares a otros centros[9].

Las Figuras obtenidas por doppler color y el análi-

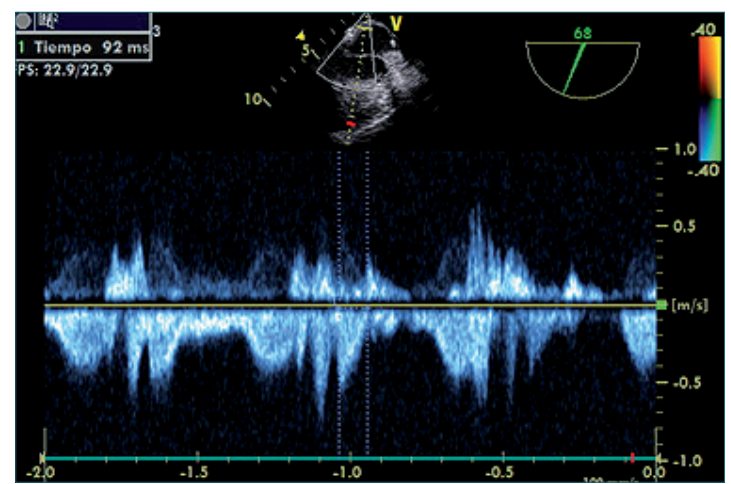

Figura 9. Tiempo de onda D hasta llegar a cero. Drenaje normal.

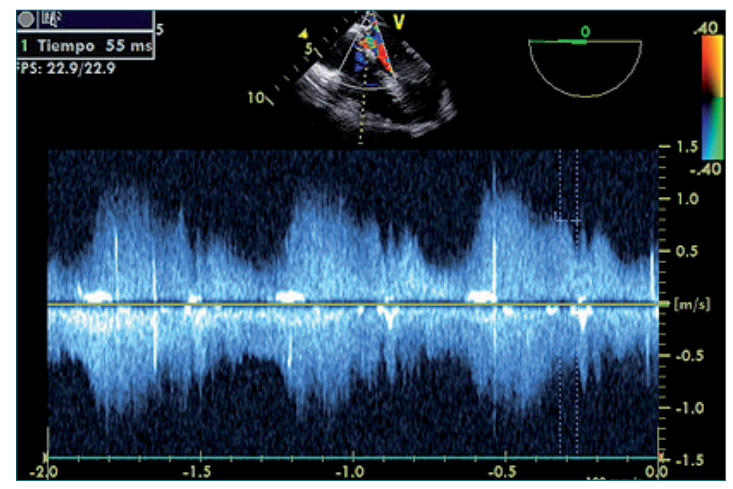

Figura 11. Tiempo de onda D hasta llegar a cero. Drenaje boca anastomótica.

sis del espectro de las ondas S, D y A de la boca anastomótica mostraron una mayor velocidad con mayor tiempo e índices S/D conservados. La importancia de observar que hay mayor aumento de tiempo y velocidad sin haber aliasing, en rangos habituales del limite nyquist de doppler color, hizo que se interpretara este fenómeno como una consecuencia de una boca anastomótica ancha. Los hallazgos ecocardiográficos certificaron una correcta reparación quirúrgica, es decir, un flujo venoso de drenaje adecuado, que se correlacionaron con la buena evolución clínica.

El paciente luego de la cirugía permaneció intubado 12 horas. Fue dado de alta a la semana posterior sin ninguna alteración en los niveles exámenes complementarios, radiografía de tórax, ni clínica. Por esto mismo se puede afirmar que las diferencias en el flujo del lado obtenidas, no fueron de implicancia clínica. El paciente regresó a controles posoperatorios semanales con excelente evolución. 


\section{Limitaciones}

Si bien podía observarse el flujo adecuado de la reparación (Figura 6), no pudo obtenerse la alineación totalmente paralela. Las venas pulmonares izquierdas no pudieron obtenerse adecuadamente, por lo que no se pudo confiar en el valor obtenido.

\section{Conclusiones}

El trabajo en equipo es fundamental en la cirugía cardiovascular. El rol del anestesiólogo ha evolucionado hacia una valoración hemodinámica más completa. En cardiopatías congénitas no sólo es importante estimar la función y la volemia, sino también corrobo- rar que cortocircuitos o defectos residuales persisten e impiden la correcta estabilidad hemodinámica, tanto en el postoperatorio inmediato como el mediato. El ecocardiograma transesofágico intraoperatorio otorga herramientas para tal fin y orienta al cirujano sobre el estado anatomofisiológico de la corrección. Tal fue este caso donde se demostró que el ecocardiograma transesofágico pudo informar que la reparación fue exitosa y sin consecuencias (como por ejemplo estenosis de la desembocadura), y así también aportó datos importantes como velocidad de venas pulmonares la cual ayudó a predecir la buena evolución y destete precoz de la ARM.

Consentimiento informado: Se adjunta el consentimiento de la madre del paciente.

\section{Referencias}

1. Dean B. Andropoulos, Stephen S, Emad B, Wanda C. Anesthesia for congenital Herat disease third edition. Capítulo 16; páginas 364-365.

2. Cooper G. Case of malformation of thoracic viscera consisting of imperfect development of the right lung and transposition of the heart. London: Med. Gazette; 1836. pp. 600-1.

3. Pablo BH, Martínez FA, de Cimitarra S. Rev Am Med Respir. 2014;4:496-487.

4. Brizuela Y, Bechara J, Jamaica L,
Dies P, Motiño L. Síndrome de la Cimitarra: un caso interesante. Bol Méd Hosp Infant México. 2011;68(6):451-4.

5. Neill CA, Ferencz C, Sabiston DC, Sheldon $\mathrm{H}$. The familial occurrence of hypoplastic right lung with systemic arterial supply and venous drainage "scimitar syndrome". Bull Johns Hopkins Hosp. 1960 Jul;107:1-21.

6. González C, Bechara J, Sáenz Gómez J, Siegert Olivares A, Jamaica Balderas L. Síndrome de cimitarra: serie de casos. Noviembre 2014. Boletín médico del hospital infantil de México.
Bol Méd Hosp Infant México. 2014;71(6):367-72.

7. Viegas C. Editorial Síndrome cimitarra. Rev Argent Cardiol. 2005 May-Jun;73(3).

8. Buller Viqueira $\mathrm{E}$, Soler Cifuentes G. y Soler Cifuentes D. Síndrome de la cimitarra. rev clín med fam 2015; 8(3): 254-256.

9. Vladimiro L. Vida, MD, PhD; Massimo A. Padalino, MD, PhD; Giovanna Boccuzzo. Scimitar Syndrome. A European Congenital Heart Surgeons Association (ECHSA) Multicentric Study. Circulation. 2010;122(12):115966. 